\title{
Control Strategy for Power Loss Reduction considering Load Variation with Large Penetration of Distributed Generation
}

\author{
Chang Liu, Xiangyu Lv, Li Guo, Lixia Cai, and Kuo Su \\ State Grid Jilin Province Electric Power Research Institute, 4433 Renmin Street, Changchun, Jilin Province, China \\ Correspondence should be addressed to Chang Liu; liuchangz0927@126.com
}

Received 17 August 2016; Accepted 8 December 2016; Published 15 February 2017

Academic Editor: Mamun B. Ibne Reaz

Copyright (C) 2017 Chang Liu et al. This is an open access article distributed under the Creative Commons Attribution License, which permits unrestricted use, distribution, and reproduction in any medium, provided the original work is properly cited.

\begin{abstract}
With the increase of penetration of distribution in distribution systems, the problems of power loss increase and short-circuit capacity beyond the rated capacity of the circuit breaker will become more serious. In this paper, a methodology (modified BPSO) is presented for network reconfiguration which is based on the hybrid approach of Tabu search and BPSO algorithms to prevent the local convergence and to decrease the calculation time using double fitness to consider the constraints. Moreover, an average load simulated method (ALS method) considering load variation is proposed such that the average load value is used instead of the actual load for calculation. Finally, from a case study, the results of simulation certify that the approaches will decrease drastically the losses and improve the voltage profiles obviously; at the same time, the short-circuit capacity is also decreased into smaller shut-off capacity of the circuit breaker. The power losses will not be increased too much even if the short-circuit capacity constraint is considered; voltage profiles are better with the constraint of short-circuit capacity considered. The ALS method is simple and the calculation time is fast.
\end{abstract}

\section{Introduction}

With the large amount of penetration of DGs, reverse power may occur and the quantity of reverse power flow will be also different with the load variation. This will make the power losses change more, whereas it is necessary to consider the load variation during the losses optimization calculation. It has been also discussed that network reconfiguration methods described in previous papers [1-3] are effective in power distribution systems.

Regarding network reconfiguration for loss minimization, one of the first papers published in this field was presented by Merlin and Back [4], who developed a heuristic approach. This solution scheme starts with a total meshed system in which all the switching elements are closed. They are then opened one by one until all the closed circuits are eliminated, and a radial system is obtained. Taking into account load variation, Chen and Cho [5] showed hourly reconfiguration benefits based on short- and long-term loss reduction. An optimal power flow model for minimal losses is applied by Shin [6]. This paper presents only results and conclusions about hourly reconfiguration for online power operation in an energy control center. Tzeng et al. [7] presented an algorithm for minimal loss reconfiguration, based on the dynamic programming approach considering the load variation.

One conclusion in common of these previous works is that they focus on the improvement of network reconfiguration algorithm, not the load model characteristics during network reconfiguration. For loss minimization calculation for a time based on network reconfiguration, if the method of network configuration changed at unit time is used, it may make the switches change frequently; although the results of losses minimization are the best, for example, for daily load, it will perform 24 operation modes in a day. To avoid this case, in the paper, the method to divide the fluctuant load into several stages is presented. The network configuration is the same at any time in the same stage, and the configuration is built based on the sum of losses minimization in each stage and short-circuit capacity reduction by the network reconfiguration method. 
Average load simulated method (ALS method) is proposed for calculation during network reconfiguration considering load variation. It will be illustrated in the next sections in detail.

\section{Approach for Loss Minimization and Short-Circuit Capacity Reduction}

2.1. Problem Formulation. Mathematically, the problem can be formulated as follows:

Objective

$$
\text { Minimize } f(X)=P_{\text {loss }}=\sum_{k=1}^{N_{s}} \sum_{i=T_{s k}}^{T_{e k}} \sum_{j=1}^{N_{b}} k_{i j} I_{i j}^{2} r_{j},
$$

where $I_{i j}$ is the current of branch $j$ at $i$ oclock, $r_{j}$ is the resistance of branch $j, N_{b}$ is the number of branches in the system, $N_{s}$ is the number of divided stages, $X$ is the switch status array, and $k_{i j}$ is the switch status of branch $j$ at $i$ o'clock; if branch $j$ is energized, $k_{i j}=1$; else, $k_{i j}=0 . T_{s k}$ and $T_{e k}$ are the start and end time point in stage $k$.

\section{Constraints}

Power flow equations:

$$
\begin{aligned}
& P_{G i}-P_{L i} \\
& \quad-V_{i} \sum_{k=1}^{N} v_{k}\left\{G_{i k} \cos \left(\theta_{i}-\theta_{k}\right)+B_{i k} \sin \left(\theta_{i}-\theta_{k}\right)\right\}=0, \\
& Q_{G i}-Q_{L i} \\
& \quad-V_{i} \sum_{k=1}^{N} v_{k}\left\{G_{i k} \sin \left(\theta_{i}-\theta_{k}\right)-B_{i k} \cos \left(\theta_{i}-\theta_{k}\right)\right\}=0 .
\end{aligned}
$$

Voltage limit:

$$
V_{i j \min } \leq V_{i j} \leq V_{i j \max }
$$

Switch status:

$$
\text { SWi }= \begin{cases}0 & \text { (if switch } i \text { is open }) \\ 1 & \text { (if switch } i \text { is closed). }\end{cases}
$$

Branches capacity limit:

$$
I_{i j}<I_{b \max }
$$

Short-circuit capacity limit:

$$
\mathrm{SCC}_{i}<\mathrm{SCC}_{\max }
$$

Radial distribution system:

$$
\begin{aligned}
N e b & =N-1, \\
\operatorname{rank}(A) & =N-1,
\end{aligned}
$$

where $N$ is the number of nodes, $V_{i j \min }$ and $V_{i j \max }$ are the maximum and minimum voltage limit of node $j$ at $i$ oclock, $I_{b \max }$ is the maximum of branch current, $\mathrm{DT}_{i}$ is the status of time point $i, \mathrm{SCC}_{\max }$ is the maximum of SCC, $\operatorname{rank}(A)$ is the row rank of branch-to-node incidence matrix $A$, and Neb is the number of energized branches.

2.2. Reconfiguration Algorithm for Power Loss for Minimum and SCC Reduction Based on MBPSO. In the paper, Modified Binary Particle Swarm Optimization (BPSO) is used for switches optimal combination. BPSO is an optimization method of discrete problem based on PSO and was proposed by Kennedy and Eberhart in 1997 [8]. BPSO has fewer parameters and is able to reach convergence fast; moreover, the result is not affected by the initial state value. The demerit of BPSO is that it easily leads to local convergence. Tabu search algorithm is able to prevent the fitness from local convergence $[9,10]$. To avoid local convergence, Tabu search algorithm and BPSO are hybrid to be used to find the gbest in this paper. In detail, if the value of gbest is invariable and the number of times for this gbest is over the setting threshold value, this gbest will be considered as the initial state and, at the same time, Tabu search algorithm will begin. Furthermore, during the searching process, once the fitness of gbest is better than the initial value (gbest), the Tabu search process will be terminated. The above measures are performed and the proposed method is called the modified BPSO (MBPSO).

The constraints must be dealt with especially because the initial BPSO does not take them into account. The equation constraints will be satisfied when the network is configured and power flow is calculated. In this paper, to dispose the inequation constraints, double fitness will be formed; namely, one is the loss minimized fitness and the other is the constraint fitness which is defined according to [11]. Furthermore, the constraint fitness will take priority. It is certain that it converges quickly into the solution zone.

2.3. Average Load Simulated Method. Average load simulated method (ALS method) is a method where the average load value is used instead of the actual load for calculation. In detail, the daily load is divided into several stages according to different loads (such as light, normal, and heavy load stage), and then the average load ratio of every stage is calculated; afterwards, network reconfiguration is done, and the optimal switches combination is got by the MBPSO algorithm. However, the fitness of switch status used to update pbest and gbset of the particle is the losses, which is calculated by the average value of all loads in a stage. Therefore, the calculation time of network reconfiguration in a stage can be decreased. Finally, the hourly loss under optimal network configuration is calculated once more by using the actual load.

2.4. Algorithm of the Proposed Method. The algorithm of ALS method for loss minimization considering SCC reduction and load variation can be described in detail as follows:

(1) Input the data and initial parameters, for example, the impedance of branches, active and reactive power of loads, the number $\left(N_{s}\right)$ of divided stages, and number of particles. 


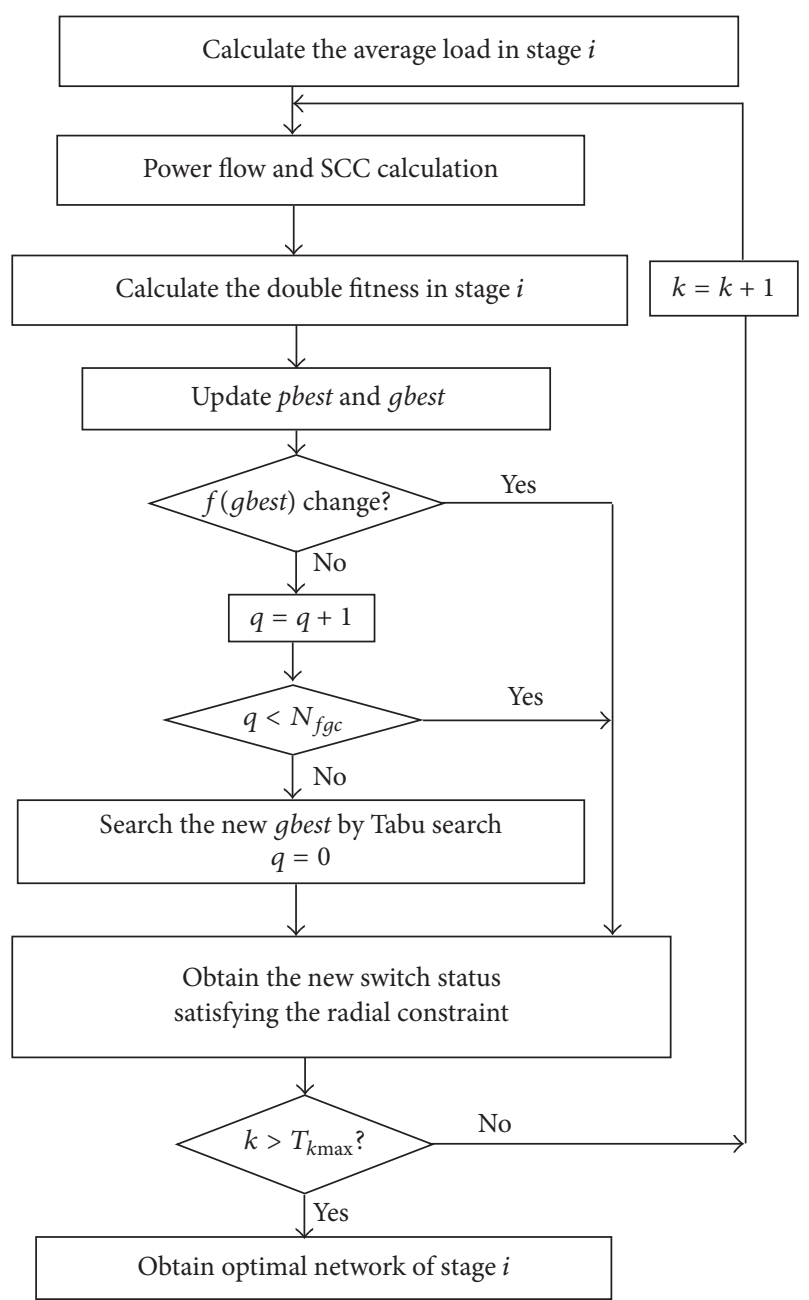

FIGURE 1: Flowchart of network reconfiguration based on average load.

(2) Iteration of optimal divided time begins; confirm the divided stages number of each particle.

(3) Get the optimal network in stage $i$ using the average load as described above and the flowchart is shown in Figure 1.

(4) $i=i+1$; then go to step 3 .

(5) Continue until termination criterion $\left(i>N_{s}\right)$ is satisfied.

(6) Obtain the optimal network in each stage and then calculate the hourly loss using the actual load.

(7) Put out the gbest, which includes the loss minimum in a day, the optimal switch combination in each time stage, and the optimal divided time mode in a day.

The flowchart of finding the optimal network by network reconfiguration is based on MBPSO in a stage. Figure 1 is the flowchart of finding the optimal network by network reconfiguration based on modified BPSO and average load in a stage. The whole flowchart of ALS method is shown in Figure 2.

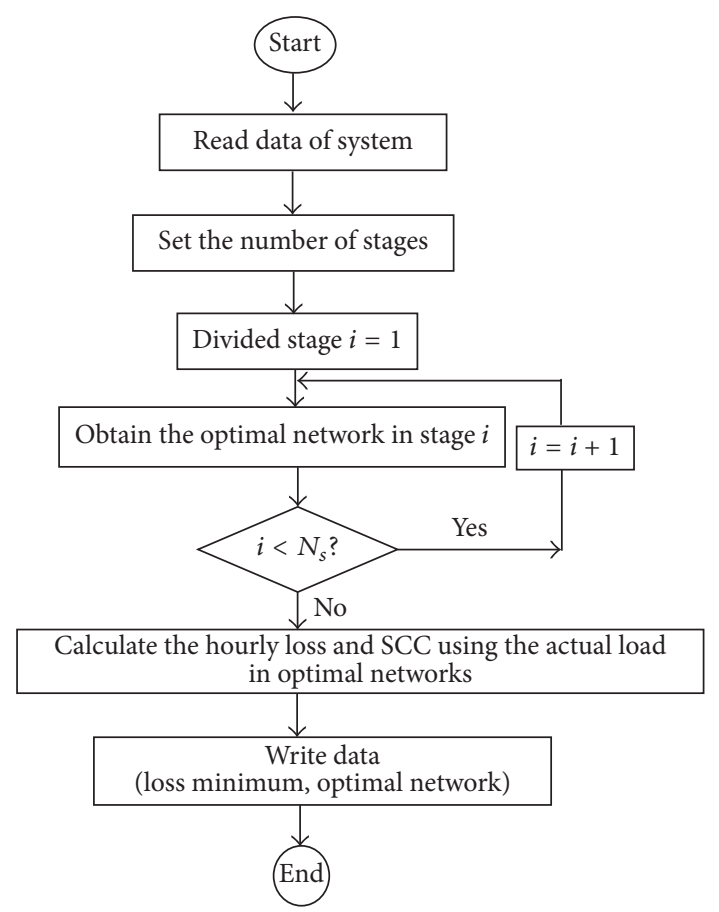

FIGURE 2: Flowchart of average load simulated method.

\section{Case Study}

Based on the configuration of [12], the data of test system is modified. The test system is a $6.6 \mathrm{kV}$ distribution system with 33 nodes and 37 branches (including 5 tie branches). The peak load includes 6.17 MW active power and 3.96 MVar reactive power. The initial network configuration is shown in Figure 1. In the figure, solid lines represent the branches in service and with closed switches; dashed lines represent the cut-off branches with open switches. The numbers 0 32 represent nodes $0 \sim 32$ and the number with " $\mathrm{S}$ " represents line switch, respectively. In the test system, the maximum of short-circuit current through CB is the one through CB1 as $\mathrm{CB1}$ is near to the upper system and all of the fault currents from generators flow through it. Hence, the 3-phase short circuit is supposed to occur at the outlet of CB1 (lines side) in location A (Figure 3). The maximum of interrupting capacity of CB1 is also considered as the constraint of SCC during simulation.

The data of system constants is given in Table 1 .

DG units are rotary generators. In this case study, the DG is the decentralized type and data is given in Table 2 .

In the following simulations, the load variation will be considered when loss and SCC are calculated. The daily load curve in summer used for simulation and data is from [11].

For the decentralized type of DG, to compare with the results of ALS method, the following three cases are considered: Case I is where the daily load is divided into three stages; Case II is where two stages are divided; and Case III is where there is only one stage in a day. The images of three cases are shown in Figure 4. 
TABLE 1: System constants.

\begin{tabular}{lccccccccc}
\hline$S_{b}$ & $V_{b}$ & $V_{\min }$ & $V_{\max }$ & SCC $_{\max }$ & $\omega_{\max }$ & $\omega_{\min }$ & $T_{k \max }$ & C1, C2 & Number of particles \\
\hline $1 \mathrm{MVA}$ & $6.6 \mathrm{kV}$ & 0.95 p.u. & 1.05 p.u. & 143 p.u. & 0.9 & 0.4 & 50 & 2 & 100 \\
\hline
\end{tabular}

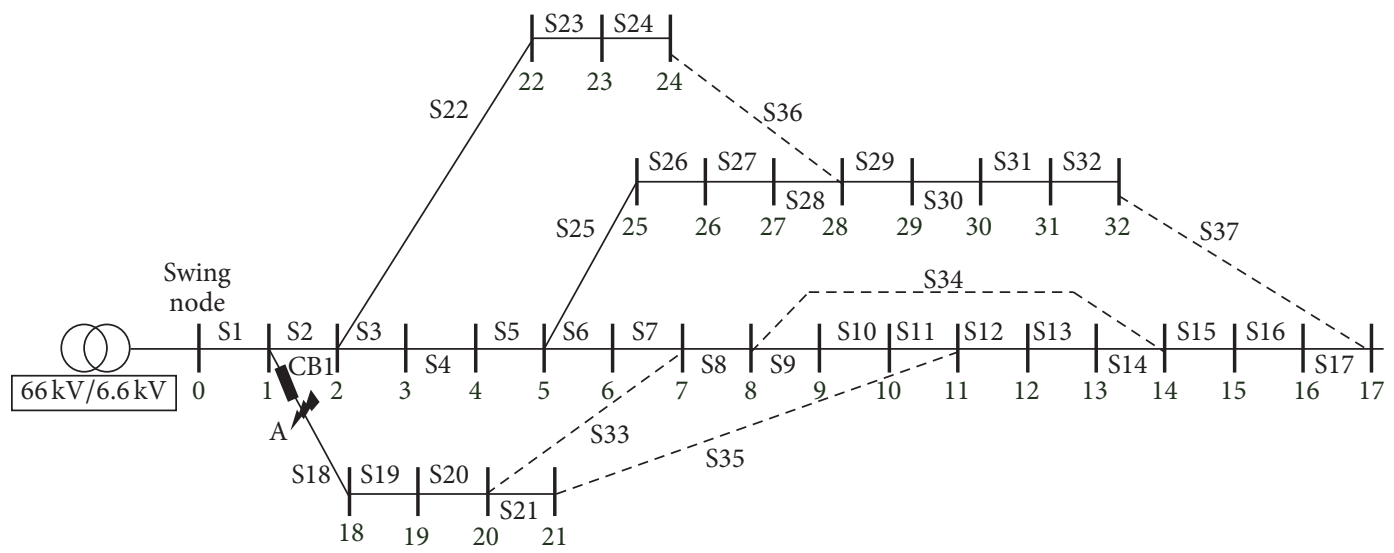

FIGURE 3: Initial network configuration of test system.

TABLE 2: Data of DG.

\begin{tabular}{lcccc}
\hline DG & Installed node & $P$ (p.u.) & $Q$ (p.u.) & $x_{d}^{\prime \prime}$ (p.u.) \\
\hline Decentralized type & $2 \sim 17,22 \sim 24$ & 0.3 & 0.185 & 0.161 \\
\hline Note. $x^{\prime \prime}$ : the subtransient reactance of $D G$ relative to its capacity
\end{tabular}

Note. $x_{d}^{\prime \prime}$ : the subtransient reactance of DG relative to its capacity.

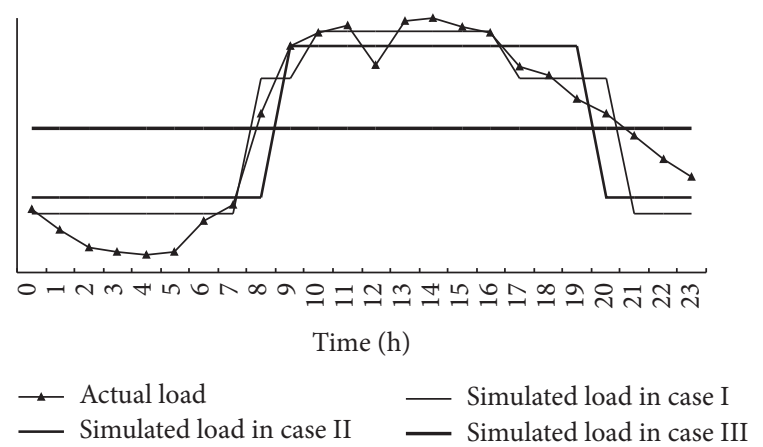

FIGURE 4: Simulated load curves of three cases.

3.1. Comparison with Different Optimal Methods. In this section, to confirm the effects of modified BPSO method on convergence and precision of calculated results, the BPSO and Tabu search methods are also done for the test system with decentralized DGs. The losses minimum of each method during iteration is shown in Figure 5.

In Figure 5, TS1 and TS2 are the results of Tabu search using the different initial configurations during iteration. The optimal values of losses for modified BPSO, BPSO, TS1, and TS2 are $96.3 \mathrm{~kW}, 101.1 \mathrm{~kW}, 98 \mathrm{~kW}$, and $105 \mathrm{~kW}$, respectively. Furthermore, the convergence of modified BPSO is also the fastest, about 13 times, and other methods are over 20 times. Therefore, it is known that the proposed method can get the optimal results fast and accurately.
TABLE 3: Data of average load in 3 cases.

\begin{tabular}{lcccccc}
\hline & \multicolumn{3}{c}{ Case I } & \multicolumn{4}{c}{ Case II } & \multirow{2}{*}{ Case III } \\
& Light & Normal & Heavy & Low & High & \\
\hline DR & $<0.75$ & {$[0.75,0.9]$} & $>0.9$ & $\leq 0.65$ & $>0.65$ & - \\
AL & 0.64 & 0.79 & 0.94 & 0.57 & 0.87 & 0.74 \\
DT & & $8,17,23$ o'clock & & 7,23 o'clock & - \\
\hline
\end{tabular}

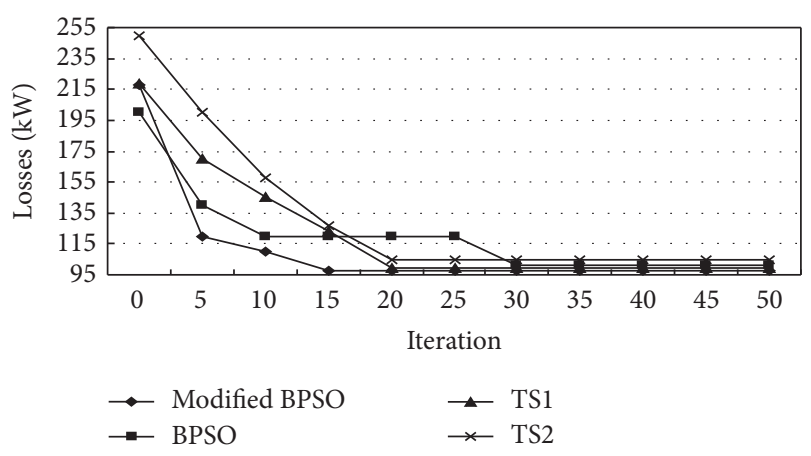

FIGURE 5: Losses minimum during iteration.

3.2. Results of Simulation. Table 3 shows the values of divided criterion (DR), average load (AL), and divided time (DT) in each stage for 3 cases.

This time, the network reconfiguration of ALS method is performed for each stage in the 3 cases by using load data shown in Figure 4. The optimal combination of switches is shown in Table 4. The loss and SCC1 at any time are calculated using optimal networks and the results are shown in Table 5. From the two tables, the conclusions of losses reduced and short-circuit capacity decreased can be understood. The calculation time is about 1 hour and decreased by a wide margin. 
TABLE 4: Open switches in each stage for 3 cases.

\begin{tabular}{cc}
\hline & Open switches in normal \\
\hline Case I & \\
Stage 1 & $\mathrm{~S} 9, \mathrm{~S} 13, \mathrm{~S} 23, \mathrm{~S} 28, \mathrm{~S} 33$ \\
Stage 2 & $\mathrm{S} 5, \mathrm{~S} 10, \mathrm{~S} 14, \mathrm{~S} 33, \mathrm{~S} 34$ \\
Stage 3 & $\mathrm{S} 4, \mathrm{~S} 10, \mathrm{~S} 13, \mathrm{~S} 33, \mathrm{~S} 34$ \\
\hline Case II & \\
Stage 1 & $\mathrm{S} 7, \mathrm{~S} 10, \mathrm{~S} 13, \mathrm{~S} 22, \mathrm{~S} 34$ \\
Stage 2 & $\mathrm{S} 5, \mathrm{~S} 10, \mathrm{~S} 14, \mathrm{~S} 33, \mathrm{~S} 34$ \\
\hline Case III & - \\
\hline
\end{tabular}

TABLE 5: Optimization results (ALS method).

\begin{tabular}{lcccc}
\hline & TCTOS & SCC1 (MVA) & Loss (kWh) & LRR \\
\hline Case I & 20 & $140.7 \sim 142.3$ & 1319.3 & $70.9 \%$ \\
Case II & 12 & $138.6 \sim 142.3$ & 1324.2 & $70.8 \%$ \\
Case III & - & - & - & - \\
\hline
\end{tabular}

Note. TCTOS is the number of total changing times of switch; LRR is power loss reduced ratio to the loss of initial network with DGs.

However, in Case III, the results could not be obtained. Voltages are over the limitation at some nodes for the actual load in the conditions of the optimal network obtained by average load. The reason is considered as follows: the optimal network is obtained based on average load, during reconfiguration, in order to minimize the losses; the margin of constraint variable to the limitation is small. If the value of actual load in a stage is much bigger or smaller than the value of average load, calculation results using actual load may be over the constraint limitation. The differential of loads in the same stage for ALS method is small as far as possible.

\section{Conclusion}

As we know, when large penetration of DGs is connected to the distribution system, the power losses may be increased and it happens probably that the short-circuit current through some circuit breakers is over the interrupting capacity of $\mathrm{CB}$ in fault. From the discussion above, the characteristics of average load simulated method (ALS method) proposed can be summarized as follows.

In the paper, network reconfiguration based on MBPSO algorithm is used for power losses minimization and shortcircuit capacity. Approaches for losses minimization and short-circuit capacity reduction are proposed considering load variation. MBPSO algorithm uses double fitness to consider the constraints, and it can decrease the calculation time.

The power losses will not be increased too much even if the short-circuit capacity constraint is considered; voltage profiles are better with the constraint of short-circuit capacity considered.

Considering the impacts of load variation on the losses, on account of the model of load used for the network reconfiguration, average load simulated method (ALS method) is approved. The approach is to divide the loads into stages and network reconfiguration in each stage is done to minimize the losses and reduce the short-circuit capacity, finally getting the minimum of losses in the whole interval time. The results of simulation certify that the approaches will decrease drastically the losses and improve the voltage profiles obviously; at the same time, the short-circuit capacity is also decreased into smaller shut-off capacity of the circuit breaker.

The ALS method is simple and the calculation time is fast; however, it is strict with the loads in the same stage.

In the current research, approaches for short-circuit capacity and power losses reduction are on the standpoint of supply system side, at the standpoint of demand side. In the following research, an approach of power losses and shortcircuit capacity on the standpoint of demand side will be presented.

\section{Competing Interests}

The authors declare that they have no competing interests.

\section{References}

[1] K. L. Butler, N. D. R. Sarma, and V. Ragendra Prasad, "Network reconfiguration for service restoration in shipboard power distribution systems," IEEE Transactions on Power Systems, vol. 16, no. 4, pp. 653-661, 2001.

[2] C. A. Dortolina and R. Nadira, "The loss that is unknown is no loss at all: a top-down/bottom-up approach for estimating distribution losses," IEEE Transactions on Power Systems, vol. 20, no. 2, pp. 1119-1125, 2005.

[3] H. Li, W. Mao, A. Zhang, and C. Li, "An improved distribution network reconfiguration method based on minimum spanning tree algorithm and heuristic rules," International Journal of Electrical Power and Energy Systems, vol. 82, pp. 466-473, 2016.

[4] A. Merlin and H. Back, "Search for a minimum-loss operating spanning tree configuration for an urban power distribution system," in Proceedings of the 5th Power Systems Computation Conference (PSCC '75), pp. 1-18, Cambridge, UK, September 1975.

[5] C. S. Chen and M. Y. Cho, "Energy loss reduction by critical switches," IEEE Transactions on Power Delivery, vol. 8, no. 3, pp. 1246-1253, 1993.

[6] B. C. G. Shin, "Development of the loss minimization function for real time power system operations: a new tool," IEEE Transactions on Power Systems, vol. 9, no. 4, pp. 2028-2034, 1994.

[7] Y.-M. Tzeng, Y.-L. Ke, and M.-S. Kang, "Generic switching actions of distribution system operation using dynamic programming method," in Proceedings of the IEEE Industrial and Commercial Power Systems Technical Conference, Detroit, Mich, USA, May 2006.

[8] J. Kennedy and R. C. Eberhart, "A discrete binary version of the particle swarm optimization," in Proceedings of the IEEE International Conference on Systems, Man and Cybernetics, vol. 5, pp. 4101-4108, Orlando, Fla, USA, 1997.

[9] D. F. Pires, A. G. Martins, and C. H. Antunes, "A multiobjective model for VAR planning in radial distribution networks based on Tabu search," IEEE Transactions on Power Systems, vol. 20, no. 2, pp. 1089-1094, 2005. 
[10] F. Tang, H. Zhou, Q. Wu, H. Qin, J. Jia, and K. Guo, "A tabu search algorithm for the power system islanding problem," Energies, vol. 8, no. 10, pp. 11315-11341, 2015.

[11] C. Liu, T. Tsuji, and T. Oyama, "Power loss minimization considering short-circuit capacity in distribution system with decentralized distributed generation," IEEJ Transactions on Electrical and Electronic Engineering, vol. 7, no. 5, pp. 471-477, 2012.

[12] M. E. Baran and F. F. Wu, "Network reconfiguration in distribution systems for loss reduction and load balancing," IEEE Transactions on Power Delivery, vol. 4, no. 2, pp. 1401-1407, 1989. 


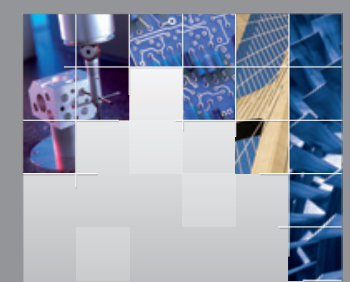

\section{Enfincering}
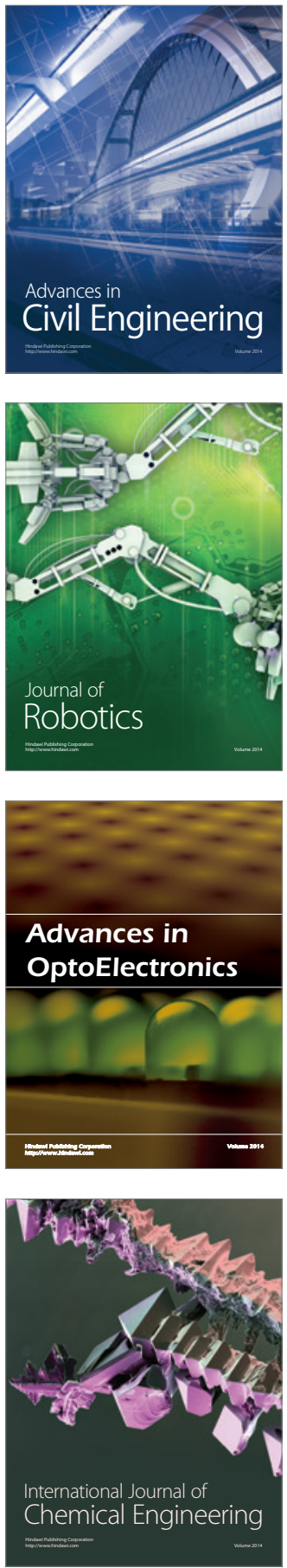

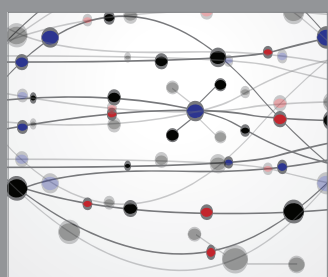

The Scientific World Journal

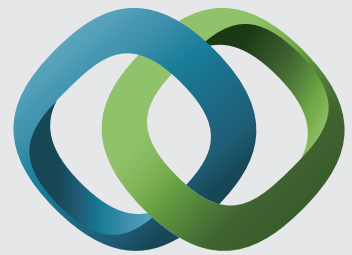

\section{Hindawi}

Submit your manuscripts at

https://www.hindawi.com
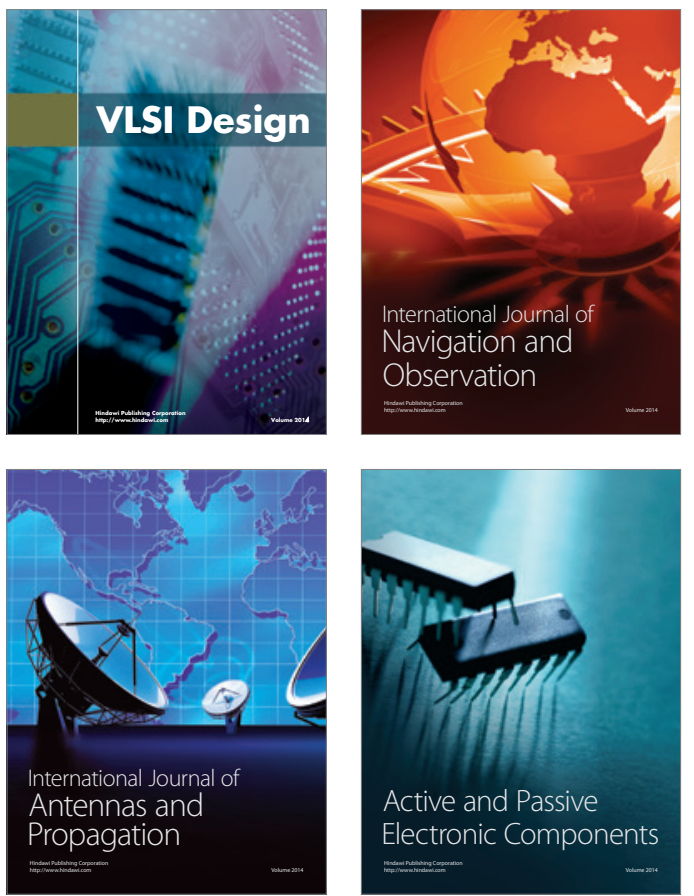
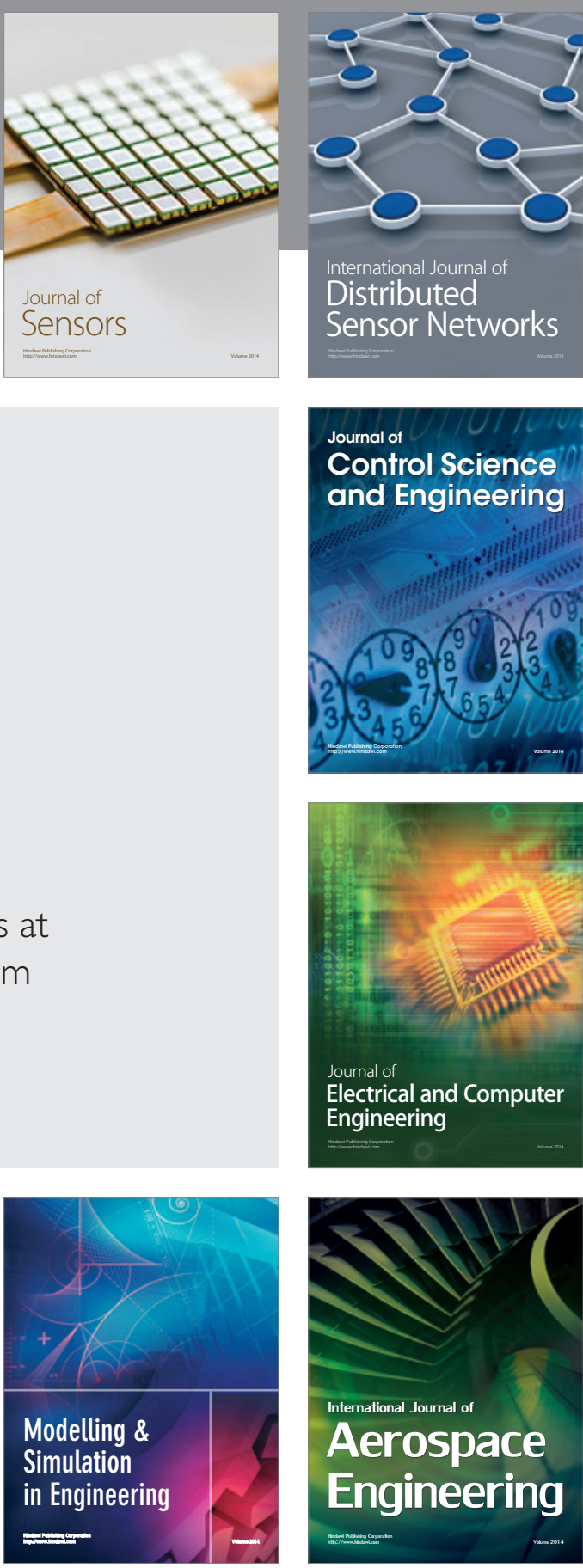

International Journal of

Distributed

Sensor Networks

$-$

Joumal of

Control Science

and Engineering
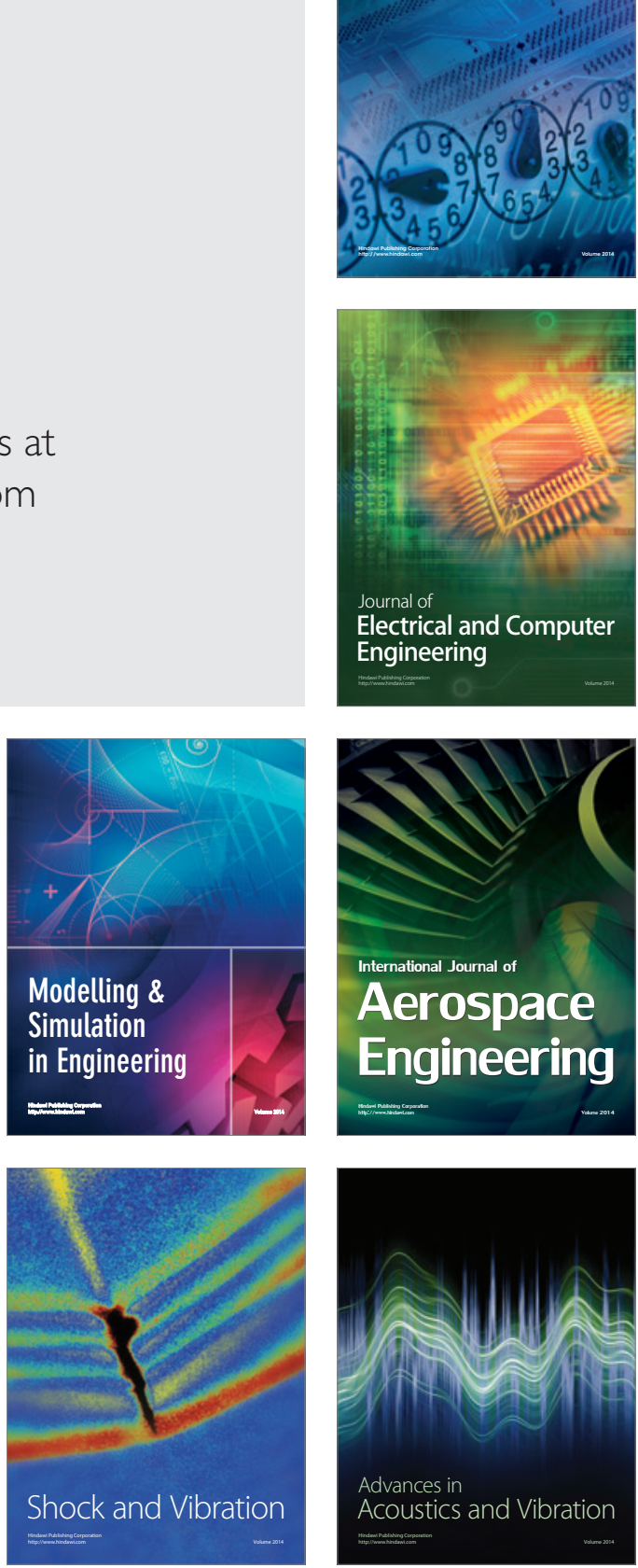